\title{
Changing Landscape of Dysglycemia Management in Critically III Patients
}

Sarvin Sanaie $1^{1^{*}}$

1. Lung and Tuberculosis Research Center, Tabriz University of Medical Sciences, Tabriz, Iran

At present, the body of evidence for blood glucose management in critically ill adults and children is not available beyond the point of proof of efficacy. Intensive blood glucose management did not pass the milestones of effectiveness and efficiency, because the multicenter trials could not confirm the results of the single-center studies. A recent review on the glucose management in critically ill adults and children suggests that use of any drug other than insulin for glucose control in intensive care unit is not recommended (1).

As we know, the reason for hyperglycemia is increasing counterregulatory hormones and insulin resistance in the target organs. Thus, in some patients who require high doses of insulin for maintenance of normoglycemia, some concerns such as hypoglycemia could be expected. Additionally, insulin therapy can be associated with hypokalemia and hypomagnesaemia which both promote insulin resistance and higher blood glucose levels. Consequently, administration of further insulin is unavoidable which in turn initiates a vicious cycle with adverse outcomes. One of therapeutic options in these situations is using insulin sensitizing agents as an adjunct therapy for glycemic control in critically ill patients. Different studies have proven that metformin, similar to insulin, is of anti-inflammatory and antioxidant properties, improves lipid profile, decreases nursing workload and lowers the incidence of adverse effects related to highdose insulin therapy without increased risk of lactic acidosis and hypoglycemia (2-4). Therefore, in patients with refractoriness to insulin who require higher doses of insulin, we might consider metformin as a safe adjunct therapy to reach targeted glucose levels.

Another important concern about glucose control in critically ill patients is accuracy of glucose measurement in these patients. The gold standard for blood glucose measurement is performed in a central hospital laboratory with hexokinase or glucose oxidase enzymatic reactions. Hence, for blood glucose management in the ICU, these tests cannot be performed and compromises inevitably have to be made. Many studies recommend that use of capillary instead of arterial blood for blood glucose measurements further amplifies the inaccuracy of blood glucose meters. Based on a meta-analysis, blood glucose measurements in

\section{Corresponding author:}

Sarvin Sanaie

Lung and Tuberculosis Research Center, Tabriz University of Medical Sciences, Tabriz, Iran

E-mail: sarvin_so2000@yahoo.com Phone: +989143116744

Receive date: 2015-06-06 | Accept date: 2015-06-25| Publish date: 2015-08-04

DOI: 10.7575/aiac.abcmed.15.03.04.02

\section{AII}


arterial blood on blood gas analyzers are more accurate than in capillary blood on blood glucose meters (5). Accordingly, drawing samples from an indwelling arterial line is the method of choice for frequent blood analysis in adult critical care areas. Sodium chloride $0.9 \%$ is the recommended flush solution for maintaining the patency of arterial catheters, but it can be easily confused with glucosecontaining bags on rapid visual examination. Unintentional use of a glucose-containing solution results in overestimation of blood glucose concentrations, leads to overtreatment with insulin and finally induces hypoglycaemia and fatal neuroglycopenic brain injury (6). As it remains a common error for incorrect fluids to be administered as arterial line flush infusions, adherence to guidelines about glucose monitoring and insulin administration is necessary for glycemic control in the presence of arterial lines as a standard method for blood sampling during insulin therapy for critically ill patients.

Based on mentioned sentences, standardization of the measurement of blood glucose concentrations is necessary. Furthermore, a new perception of hyperglycemia and its management should be assessed in future large trials to elucidate advantages of glucose management in these patients.

\section{References}

1-Mesotten D, Preiser J.Ch, Kosiborod M.Glucose management in critically ill adults and children. Lancet Diabetes Endocrinol 2015. Published Online June 11, 2015. http://dx.doi.org/10.1016/ S2213-8587(15)00223-5.

2-Ansari Gh, Mojtahedzadeh M, Kajbaf F, Najafi A, Khajavi MR, Khalili H, et al. How does blood glucose control with metformin influence intensive insulin protocols? Evidence for involvement of oxidative stress and inflammatory cytokines. Adv Ther. 2008; 25(7): 681-702.

3-Mojtahedzadeh M, Rouini MR, Kajbaf F, Najafi A, Ansari Gh, Gholipour A, et al. Advantage of adjunct metformin and insulin therapy in the management of glycemia in critically ill patients. Evidence for nonoccurrence of lactic acidosis and needing to parenteral metformin. Arch Med Sci 2008; 4, 2: 174-181.

4-Mojtahedzadeh M, Jafarieh A, Najafi A, Khajavi MR, KhaliliN.Comparison of metformin and insulin in the control of hyperglycaemia in non-diabetic critically ill patients. Pol J Endocrinol 2012; 63 (3): 206-211.

5- Inoue S, Egi M, Kotani J, Morita K. Accuracy of blood-glucose measurements using glucose meters and arterial blood gas analyzers in critically ill adult patients: systematic review. Crit Care 2013; 17: R48.

6- Sprint Working Party, Woodcock TE, Cook TM, Gupta KJ, Hartle A. Arterial line blood sampling: preventing hypoglycaemic brain injury 2014: the Association of Anaesthetists of Great Britain and Ireland. Anaesthesia. 2014 Apr; 69(4):380-5. doi: 10.1111/anae.12536. Epub 2014 Feb 6. 